\title{
Effects of a Required Large-Group Mindfulness Meditation Course on First-Year Medical Students' Mental Health and Quality of Life: a Randomized Controlled Trial
}

\author{
Afonso Damião Neto, MD, MSc, Alessandra Lamas Granero Lucchetti, MD, PhD, \\ Oscarina da Silva Ezequiel, MD, PhD, and Giancarlo Lucchetti, MD, PhD
}

School of Medicine, Federal University of Juiz de Fora, Juiz de Fora, MG, Brazil.

\begin{abstract}
BACKGROUND: Teaching mindfulness techniques has been used in the attempt to prevent mental health problems in medical students. Although it has already shown promising results when offered to volunteers, the use as a required strategy is still controversial.
\end{abstract}

OBJECTIVES: To verify the efficacy of teaching mindfulness techniques to large groups when made part of a required discipline at the beginning of medical training.

DESIGN: Randomized controlled trial

PARTICIPANTS: First-year medical students at the Federal University of Juiz de Fora, Brazil

METHOD: Students were randomized into two groups: an intervention group (receiving a 6-week mindfulness protocol) and a control group (given a 6-week course containing organizational aspects of the medical school).

MAIN MEASURES: Intervention and control groups were compared on the levels of quality of life (WHOQOL-Bref), stress, anxiety and depression (DASS 21) and the facets of mindfulness (FFMQ) at baseline and at the end of the intervention.

RESULTS: A total of 141 students were included in the study, 70 in the intervention group and 71 in the control group. No significant differences were found between the intervention and control groups in all mental health, quality of life, and FFMQ scores (Cohen's $d=0.01$ to 0.14 ). Likewise, no significant gains in mental health measures, quality of life, and FFMQ were identified in the intervention group when compared with the control group (Cohen's $d=0.02$ to 0.33).

CONCLUSIONS: The incorporation of a required mindfulness course for large groups in the curriculum during the first semester of medical training was not associated with an improvement on medical students' mental health and quality of life.

\section{CLINICAL TRIALS REGISTRATION: NCT03132597.}

KEY WORDS: mindfulness; meditation; medical students; quality of life; depression.

J Gen Intern Med 35(3):672-8

DOI: $10.1007 / \mathrm{s} 11606-019-05284-0$

(c) Society of General Internal Medicine 2019

Electronic supplementary material The online version of this article (https://doi.org/10.1007/s11606-019-05284-0) contains supplementary material, which is available to authorized users.

Received November 13, 2018

Revised June 14, 2019

Accepted July 25, 2019

Published online August 26, 2019

\section{INTRODUCTION}

Medical students are particularly vulnerable to symptoms of stress, anxiety, and depression. ${ }^{1-3}$ Recent studies have shown high rates of mental health problems during medical training in different parts of the world, as demonstrated in a systematic review and meta-analysis of 195 studies involving 129,123 medical students in 47 countries. In this review, $27.2 \%$ of students presented positive symptoms for depression and $11.1 \%$ demonstrated suicidal thoughts. ${ }^{4}$

Various factors are listed as possible facilitators of this condition, such as long hours working and studying, unhealthy learning environments, sleep deprivation, and factors that interfere in daily personal life. ${ }^{1,2}$ Nevertheless, these factors do not completely justify why first-year students have begun their activities already having high levels of anxiety and burnout. ${ }^{3,5-7}$

The need to deal with the unknown and expectations of beginning a new phase in their lives and needing to leave home (as do most students at our university) are possible explanations. ${ }^{3}$ We must also consider the remarkable levels of psychiatric symptoms among Brazilian adolescents in general (33.6\%), as observed in a study involving 74,589 Brazilian adolescents. ${ }^{8}$

In this context, the use of mindfulness training techniques has shown to be an effective tool for promoting mental health, leading to reduced stress, depression, and anxiety, and to improved quality of life, as demonstrated by a meta-analysis that included 29 studies $(n=2668)$ and found a moderate effect for these outcomes. ${ }^{9}$

Mindfulness is frequently described as "the awareness that emerges through paying attention on purpose, in the present moment, and nonjudgmentally to the unfolding of experience moment by moment". ${ }^{10}$ The original protocol for mindfulness-based interventions consists of teaching techniques adapted from yoga and Buddhism in a non-religious form. It is administered in 8 encounters of two and a half hours each associated with a day of withdrawal in silence (i.e., meditation silent retreat). ${ }^{11}$ Shorter protocols have also been shown to be effective in non-clinical populations. ${ }^{12}$

Specifically with medical students, in addition to using the original protocol, ${ }^{13}$ others with varied formats have been used, ${ }^{14}$ demonstrating the long-term impact of this protocol. 
A Malaysian study found improvements in the scales of mindfulness, stress, and self-efficacy ${ }^{15}$; an American study found improvements in anxiety during a 4 -week program ${ }^{16}$; a Norwegian study found improvements in well-being, anguish, and the facet of nonjudgmental mindfulness during a 7 -week protocol ${ }^{17}$; and a Tasmanian study found improvement of anxiety with an 8-week intervention done through daily practice conducted with audio CDs. ${ }^{18}$

In spite of these promising results, the studies previously described, with the exception of the Norwegian study,${ }^{17}$ have been conducted with small groups (10 to 20 students) of an elective nature. Studies are needed in larger, mandatory groups (including all students) to evaluate if they are equally effective. Likewise, there are few randomized controlled studies to verify the efficacy of teaching mindfulness techniques on medical students' mental health, particularly in Brazilian and Latin-American medical schools.

Thus, the objective of this study is to evaluate the effects of a required large-group mindfulness meditation course on firstyear medical students' mental health and quality of life, when compared with a control group.

\section{METHODS}

\section{Study Design and Participants}

This is a randomized controlled trial with an allocation ratio of 1:1, carried out from August 2016 to July 2017 including two classes of first-year incoming medical students at the Federal University of Juiz de Fora (UFJF, acronym in Portuguese): the class from the second semester of 2016 and the class from the first semester of 2017. This school is a public university with a predominantly traditional curriculum that enrolls approximately 85 to 90 students per semester (ranging from 170 to 180 medical students per year). During the beginning of the medical course, students study anatomy, biochemistry, biophysics, cellular and molecular biology, histology and embryology, heath systems, introduction to the practice of medicine, and introduction to the university life.

The study was approved by the UFJF University Hospital's Ethics in Research Committee. Students signed a consent form. The study protocol is registered at clinicaltrials.gov under the number 61240016.3.0000.5133; NCT03132597.

\section{Eligibility Criteria}

To be included, students had to be at least 18 years old and officially enrolled in the first year of medicine. Students who did not fill out all questionnaires, who did not sign the consent form, who withdrew from medical school, or who were not present when data was collected were excluded.

\section{Procedures}

Students enrolled in a required course named "Introduction to University Life," administered as a 2-h weekly class over a period of 15 weeks, were allocated into two groups: an intervention group and a control group. Both groups were informed during the course's first week about the discipline's objectives, what mindfulness is, and participation in the research.

Beginning the second week, the intervention group received a 6-week course about the practice of mindfulness while the control group was given theoretical content in which they were shown organizational aspects of medical school (library, evaluations, "being a doctor," how the medical school departments function, scholarships, and student assistance, among others) in a practical and friendly way. After the study was concluded, students in the intervention group were given the content previously administered to the control group.

To evaluate outcomes before and after the intervention, questionnaires were applied at the beginning (first week of medical school) and end of the intervention (after the sixth week of the intervention, approximately 8 weeks after beginning the school year).

\section{Intervention}

The mindfulness course was inspired by the methodology proposed by Kabat-Zinn (10) and adapted to be given over 6 weeks to large groups (i.e., 45 students) during 2-h encounters. The course was administered by one instructor (this article's first author) - a medical doctor working in the area of labor health at the institution who has practiced meditation for 8 years and has been teaching mindfulness techniques to University professors and staff for 2 years.

- Goals of the course: By the end of the course, students should be able to (a) understand the basic precepts and the evidence of mindfulness; (b) experience and have knowledge of various meditation techniques; (c) learn and develop practical skills of mindfulness meditation; (d) become aware of thoughts, surroundings, smells, bodily movements, and breath; (e) learn how to cultivate positive emotions (i.e., compassion, forgiveness, kindness); (f) calm down in moments of anger, high stress, and anxiety; and $(\mathrm{g})$ incorporate these meditation techniques into daily life activities and communication.

- Teaching format: Students stayed in a conventional classroom with school chairs. All classes were eminently practical, with the exception of the first encounter when they had a lecture in the first hour and a practical activity in the second hour. The classes usually started with an open group discussion in which students were encouraged to relate their home practice experiences. Then, the instructor explained the activities for that day and carried out practical activities involving several meditation techniques guided by the instructor (ranging from 10 to $20 \mathrm{~min}$ ). After each meditation technique, students discussed in the classroom their experiences, difficulties, and doubts. At the end of the encounter, the instructor reinforced the importance of practicing meditation at 
home and guided how the student could use these techniques in their daily life activities.

- Schedule: The schedule was structured as follows:

1.- First week - conversation about students' knowledge and experiences, the theory and evidence of mindfulness, raisin exercise (this practice aims to bring acute awareness to the senses and ultimate eating habits; the idea is that focusing the attention on the tiny raisin could help bringing the mind into the moment and train it to notice the present), and body scan (it focuses attention on physical sensations in the body, performed by "scanning" one's awareness through the entire body on a micro level and giving attention to every inch of the body).

2.- Second week - practice of nonjudgmental listening awareness, awareness of thoughts and emotions while visualizing walking down the street, greeting an acquaintance without having your greeting returned. Mountain meditation (the mountain is a metaphor for the human experience where the participant imagines his/her body becoming the mountain in order to draw on a mindful awareness of the present moment).

3.- Third week - mindfulness of breathing and practice of breathing exercises, diaphragmatic breathing, and breathing in four equal sequences (inhaling, exhaling, and the times between both).

4.- Fourth week - Handling thoughts and emotions during practices, practice visualizing challenging situations before and after using a breathing space, and walking meditation (participant learns to be aware as he/she walks, to use the natural movement of walking to cultivate mindfulness and wakeful presence).

5.- Fifth week - sitting meditation with changes in focus of attention (body, breathing, hearing, thoughts, and emotions), and loving kindness (Metta) meditation (practice stimulating the heartfelt wish for the wellbeing of oneself and others, a systematic cultivation of the capacity for loving kindness).

6.- Sixth week-Mindfulness of breathing, awareness, listing individual daily activities, and students' final impressions.

- Home practice: Students were sent four audios in MP3 format via email for guided home practice and were asked to practice daily for at least $10 \mathrm{~min}$ and bring a meditation diary including information of how many days students had practiced at home. Nevertheless, only approximately $20 \%$ of students have returned the meditation diaries back and the reason for this low rate was lack of time, forgetfulness, and lack of interest. For this reason, the meditation diary was discontinued. They were also encouraged to relate their home practice experiences at the beginning of each encounter.

\section{Instruments}

A self-reporting questionnaire lasting 20 min was administered to the medical students and included:

1. Socio-demographic data: age, ethnicity, marital status, and family income;

2. Religiousness: Duke Religion Index, made up of five items evaluating three different dimensions of religious involvement (organizational, non-organizational, and intrinsic). This scale has been validated for use in Brazil $^{19}$;

3. DASS 21-Depression, Anxiety, and Stress Scale: validated for Portuguese, it is made up of 21 items, allowing simultaneous evaluation of symptoms of depression, anxiety, and stress. Easy to apply in clinical and non-clinical environments and adequate for different age groups ${ }^{20}$;

4. WHOQOL-BREF-World Health Organization Quality of Life: validated for Portuguese. This instrument is widely used by the scientific community and has 26 items distributed in four domains: physical, psychological, social relations, and environment, and a general facet: "quality of life" and "health"21;

5. Five Facets of Mindfulness (FFMQ-BR): questionnaire made up of 39 items that evaluate five facets of mindfulness: observing, describing, acting with awareness, not judging internal experiences, and not reacting to internal experiences. This scale has also been validated for Portuguese ${ }^{22}$;

6. Impact of the mindfulness course in student's life: participants were asked whether they perceived a positive difference in their life after the mindfulness course with the possible answers "yes" or "no."

\section{Randomization}

Simple randomization was done using random.org software (computer-generated random numbers) using the list of enrolled students made available by course administrators. Allocation, as previously described, was 1:1 (1 intervention:1 control).

\section{Sample Size}

Sample size was calculated using G*Power 3.1. Based on previous studies favoring mindfulness interventions against control groups for mental distress (Cohen's $d=-0.77)^{17}$ and 
for depression (Cohen's $d=-0.54),{ }^{23}$ we found that, considering a 1 -Beta $=0.80$, alpha $=0.05$, allocation ratio $1: 1$ and two tailed, the sample size would be at least 55 participants per group, totaling 110 participants included in the final analyses.

\section{Statistical Analysis}

A descriptive analysis was carried out using sociodemographic data and the results obtained from each instrument described above. Absolute and relative frequency measurements were used for each categorical variable, as well as measurements of mean and standard deviation for continuous variables. Baseline characteristics were compared between groups (control versus intervention) using chi-square and independent $t$ tests.

To investigate whether the intervention was effective or not after 6 sessions, post-intervention scores of the DASS-21 and WHOQOL-Bref were compared between groups using independent $t$ tests. Likewise, a comparison of the changes in scores (post- and pre-measurements) between groups was also conducted using independent $t$ tests. The effect sizes $(d)$ were calculated and followed these values: 0.20 as small, 0.50 as medium, and 0.80 as large. ${ }^{24}$

Finally, correlations between quality of life, DASS-21, and FFMQ were carried out using Pearson correlation tests.

Analyses followed the intent-to-treat (ITT) principle, using the last observation carried forward method and also the "per protocol" method.

The statistical program SPSS, version 20.0 (SPSS Inc.), was used for analysis. A $p \leq 0.05$ was considered significant.

\section{RESULTS}

Of a total of 171 medical students invited to participate, 141 $(82.4 \%)$ answered the baseline questionnaire and were included in the intent-to-treat analysis, 70 in the intervention group and 71 in the control group. Excluded students were absent in the first baseline data collection.

Table 1 presents the baseline characteristics. No differences were found concerning demographics, mental health measures (DASS 21), FFMQ, and quality of life (WHOQOL), with the exception of the WHOQOL Psychological $(p=0.01)$, which favored the control group.

Post-intervention measures are shown in Table 2. No differences were found between mental health, quality of life, and FFMQ scores. Effect sizes were calculated, revealing from small to very small effects (Cohen's $d=0.01$ to 0.14 ).

Table 3 shows the changes (post- and pre-measurements) between groups and revealed that no significant gains in mental health measures and quality of life were identified in the intervention group when compared with the control group. Effect sizes were calculated and revealed from small to very small effects (Cohen's $d=0.02$ to 0.18 ). FFMQ measurements were non-significant higher in the intervention group with a
Table 1 Baseline Characteristics and Comparison Between Groups

\begin{tabular}{llll}
\hline \hline & $\begin{array}{l}\text { Intervention, } n \\
(\%)\end{array}$ & $\begin{array}{l}\text { Control, } n \\
(\%)\end{array}$ & $p$ \\
$\begin{array}{l}\text { Gender } \\
\text { Male }\end{array}$ & $34(48.6 \%)$ & $36(50.7 \%)$ & \\
$\quad$ Female & $36(51.4 \%)$ & $35(49.3 \%)$ & 0.867 \\
Ethnicity & $46(65.7 \%)$ & $41(57.7 \%)$ & \\
$\quad$ White & $24(34.3 \%)$ & $30(42.3 \%)$ & 0.330 \\
$\quad$ Non-white & & & \\
Income & $30(42.8 \%)$ & $29(40.9 \%)$ & \\
$\quad$ High & $40(57.2 \%)$ & $42(59.1 \%)$ & 0.808 \\
$\quad$ Medium/low & Mean (SD) & Mean (SD) & $p$ \\
& $18.87(1.81)$ & $19.07(1.96)$ & 0.534 \\
Age & $114.09(17.99)$ & 119.90 & 0.054 \\
FFMQ & & $(15.73)$ & \\
& $4.47(4.24)$ & $3.82(3.77)$ & 0.345 \\
DASS Depression & $4.37(3.50)$ & $3.87(3.57)$ & 0.402 \\
DASS Anxiety & $8.68(4.16)$ & $7.54(3.98)$ & 0.102 \\
DASS Stress & $14.80(2.00)$ & $14.84(2.20)$ & 0.906 \\
WHOQOL Physical & $13.79(1.89)$ & $14.63(1.87)$ & 0.011 \\
WHOQOL & & & \\
Psychological & $15.67(2.61)$ & $15.38(2.75)$ & 0.534 \\
WHOQOL Social & $15.14(2.08)$ & $14.89(2.20)$ & 0.490 \\
WHOQOL & & & \\
Environmental & & & \\
\hline
\end{tabular}

FFMQ, Five Facet Mindfulness Questionnaire; WHOQOL, World Health Organization Quality of Life questionnaire

moderate effect size favoring the intervention (Cohen's $d=$ $0.33)$.

Finally, a correlation matrix (Table 4) between FFMQ and mental health and quality of life measures was carried out showing that gains in the FFMQ were correlated to less depression, and stress, and better psychological quality of life. Concerning the satisfaction of students with the mindfulness course, $47(67.1 \%)$ answered that they have perceived a positive difference in their life after the mindfulness course.

The "per protocol" analysis is presented in the supplementary material and included $114(80.8 \%)$ out of 141 students included in the ITT, 57 in the intervention group and 57 in the control group. The reason for exclusion in the "per protocol" analysis was not answering the post-test questionnaire. The

Table 2 Post-intervention Comparison Between Groups

\begin{tabular}{lllll}
\hline \hline & $\begin{array}{l}\text { Intervention, } \\
\text { mean (SD) }\end{array}$ & $\begin{array}{l}\text { Control, } \\
\text { mean } \\
\text { (SD) }\end{array}$ & $p$ & $\begin{array}{l}\text { Cohen's } \\
\mathbf{d}^{\#}\end{array}$ \\
\hline FFMQ & $115.76(20.38)$ & $\begin{array}{l}116.30 \\
(17.04)\end{array}$ & 0.867 & 0.02 \\
DASS & $3.91(3.34)$ & $\begin{array}{l}3.97 \\
(3.28)\end{array}$ & 0.918 & 0.01 \\
Depression & $3.74(3.47)$ & $\begin{array}{l}3.57 \\
(3.45)\end{array}$ & 0.777 & 0.04 \\
DASS Anxiety & & 0.551 & 0.09 \\
DASS Stress & $8.18(4.03)$ & $\begin{array}{l}7.76 \\
(4.40)\end{array}$ & 0.953 & 0.01 \\
WHOQOL & $14.08(1.98)$ & $\begin{array}{l}14.06 \\
(2.27)\end{array}$ & 0.382 & 0.14 \\
Physical & $13.89(1.99)$ & $\begin{array}{l}14.19 \\
(2.09)\end{array}$ & 0.924 & 0.01 \\
WHOQOL \\
$\begin{array}{l}\text { Psychological } \\
\text { WHOQOL }\end{array}$ & $15.70(2.42)$ & $\begin{array}{l}15.65 \\
(2.91)\end{array}$ & 0.740 & 0.05 \\
$\begin{array}{l}\text { Social } \\
\text { WHOQOL }\end{array}$ & $15.09(2.27)$ & $\begin{array}{l}14.96 \\
(2.32)\end{array}$ & & \\
Environmental & & & & \\
\hline
\end{tabular}

FFMO, Five Facet Mindfulness Questionnaire; WHOQOL, World Health Organization Quality of Life questionnaire

${ }^{\#}$ The effect sizes (d) followed these values: 0.20 as small, 0.50 as medium, and 0.80 as large 
Table 3 Changes (Post-Pre Intervention) Between Groups

\begin{tabular}{|c|c|c|c|c|}
\hline & $\begin{array}{l}\text { Intervention, } \\
\text { mean (SD) }\end{array}$ & $\begin{array}{l}\text { Control, } \\
\text { mean } \\
\text { (SD) }\end{array}$ & $p$ & $\begin{array}{l}\text { Cohen's } \\
d^{\#}\end{array}$ \\
\hline FFMQ & $0.88(13.71)$ & $\begin{array}{l}-3.36 \\
(11.68)\end{array}$ & 0.061 & 0.33 \\
\hline $\begin{array}{l}\text { DASS } \\
\text { Depression }\end{array}$ & $-0.55(4.05)$ & $\begin{array}{l}0.17 \\
(3.62)\end{array}$ & 0.266 & 0.18 \\
\hline DASS Anxiety & $-0.57(3.05)$ & $\begin{array}{l}-0.29 \\
(3.04)\end{array}$ & 0.584 & 0.09 \\
\hline DASS Stress & $-0.40(4.09)$ & $\begin{array}{l}0.05 \\
(3.41)\end{array}$ & 0.471 & 0.11 \\
\hline $\begin{array}{l}\text { WHOQOL } \\
\text { Physical }\end{array}$ & $-0.71(1.60)$ & $\begin{array}{l}-0.75 \\
(1.66)\end{array}$ & 0.872 & 0.02 \\
\hline $\begin{array}{l}\text { WHOQOL } \\
\text { Psychological }\end{array}$ & $0.04(2.09)$ & $\begin{array}{l}-0.29 \\
(1.89)\end{array}$ & 0.325 & 0.16 \\
\hline $\begin{array}{l}\text { WHOQOL } \\
\text { Social }\end{array}$ & $-0.06(2.30)$ & $\begin{array}{l}0.29 \\
(2.45)\end{array}$ & 0.394 & 0.14 \\
\hline $\begin{array}{l}\text { WHOQOL } \\
\text { Environmental }\end{array}$ & $-0.05(1.52)$ & $\begin{array}{l}0.04 \\
(1.66)\end{array}$ & 0.730 & 0.05 \\
\hline
\end{tabular}

FFMQ, Five Facet Mindfulness Questionnaire; WHOQOL, World Health Organization Quality of Life questionnaire

${ }^{\#}$ The effect sizes (d) followed these values: 0.20 as small, 0.50 as medium, and 0.80 as large

results found in the "per protocol" analysis are better visualized in the supplementary material and are similar to those of the ITT analysis.

\section{DISCUSSION}

This study revealed no significant differences between students submitted to a required large-group mindfulness meditation protocol in relation to a control group in terms of quality of life, anxiety, stress, and depression among medical students. These results add to current medical literature by showing that, at least in the Brazilian context, the attempt to conduct mindfulness sessions for large groups (i.e., increasing the costeffectiveness of the intervention) and within the curriculum as a required item was not successful.

Mindfulness has been used with medical students for at least 20 years. ${ }^{13}$ It has demonstrated beneficial effects that have been repeatedly confirmed by later studies, independent of the format in which they were structured. ${ }^{15-18}$ Nevertheless, these studies were offered in elective courses that included individuals who were volunteers and more inclined to adhere to that intervention. In addition, most studies were quasi-

Table 4 Correlation Between FFMQ, DASS21, and WHOQOL

\begin{tabular}{ll}
\hline \hline & Delta FFMQ $(\boldsymbol{r})$ \\
\hline Delta DASS21 Depression & $-0.333^{* *}$ \\
Delta DASS 21 Anxiety & -0.153 \\
Delta DASS21 Stress & $-0.294^{* *}$ \\
Delta WHOQOL Physical & $0.191^{*}$ \\
Delta WHOQOL Psychological & $0.353^{* *}$ \\
Delta WHOQOL Social & 0.120 \\
Delta WHOQOL Environmental & 0.119
\end{tabular}

FFMQ, Five Facet Mindfulness Questionnaire; WHOQOL, World Health Organization Quality of Life questionnaire

Delta: post-pre

$r=$ Pearson's coefficient, $* p<=0.05, * * p<=0.01$ experimental without randomization or without control groups. Thus, the gains observed can be confused with the motivation and interest of those who take advantage of this opportunity. ${ }^{25}$

However, as corroborated by other studies, required courses in general have not shown to improve students' quality of life and mental health. In a quasi-experimental study, a required longitudinal mindfulness-based stress management course was added for first-year students in the USA. ${ }^{26}$ The authors did not find measurable improvements in medical student well-being or empathy. Similarly, another study conducted in Ireland compared teaching mindfulness as a required element in the first year and voluntarily in the second, resulting in greater acceptance and satisfaction for the course offered as an elective. ${ }^{27}$ These initial studies corroborate the findings of our randomized trial, showing that a required course might not be the most appropriate form for this intervention.

The conflicting findings described above could be explained by the very definition of mindfulness: "the awareness that emerges through paying attention on purpose, in the present moment, and nonjudgmentally to the unfolding of experience moment by moment", ${ }^{10}$ making any intervention based on mindfulness totally dependent on the subject's intention, acceptance, and judgment. In this way, it seems that one's mere presence in a classroom without deliberate home practice and training is innocuous. In our study, it was observed that some students had little engagement with the course and minimal interest in practice. Only a small portion of students reported that they were practicing at home.

Beyond the manner of offering the course (required or elective), the differences among countries and institutions should be taken into consideration when analyzing the results of different protocols for teaching mindfulness techniques as well as the characteristics of the personality of students submitted to the training. Student well-being depends on a complex interaction among various factors, highlighting the importance of cultural and educational influences, as seen in those found among Brazilian and American medical students. ${ }^{28}$ First-year students in Brazil are younger than those in the USA and tend to be exceedingly concerned about grades and evaluations. In the same way, students' intrinsic characteristics can also influence success of the intervention. Previous studies with medical and psychology students in Norway have shown that the mindfulness intervention has greater effect on individuals with psychological characteristics of greater conscientiousness and neuroticism. ${ }^{29}$ Another factor that can be associated with the lack of positive findings is the use of large groups (45 students), different from the small groups (between 10 and 20 individuals) used in previous studies. ${ }^{13,15,16,30}$

Our study also found a correlation between the mindfulness scale and the quality of life, anxiety, stress, and depression scales. The relation between reduced stress and increased characteristics of mindfulness was made evident in previous studies $(r=-0.42, p=0.01),{ }^{16}$ suggesting that an increase in 
the state of mindfulness would be a good way to promote mental health in those medical students who dedicate themselves to the course. Nevertheless, it is generally perceived that the intervention did not lead to an increase in the FFMQ, reinforcing the idea that not all students dedicated themselves to the course.

The rigorous competition in medical school, the intense routine, the study overload, and the great expectations carried by students' relatives bring about the need for interventions that could potentially improve the burnout and the mental health of these students. The addition of mindfulness as a required activity for many students during the first period has become just one more task, different from the intellectual ones to which they are accustomed and without apparent palpable and immediate results. We observed that mindfulness in a required course did not prove useful in promoting the mental health in first-year medical students.

The timing of this type of curriculum is important and this could potentially influence the results. As related earlier, firstyear students in Brazil tend to be younger, which can lead them to a certain level of indifference about the course. Perhaps a course offered during clerkship, a period where stress is higher and students have more clinical tasks and contact with patients, would have better outcomes. Instead of a single curriculum exposure for a short period of time, a longitudinal long-term approach would be preferable and could be more successful, acting as a preventive but also as a therapeutic intervention. Nevertheless, there is a lack of space in the curriculum which hinders these initiatives in most institutions. A good strategy would be to cultivate a "culture of mindfulness" in the institution, ${ }^{31}$ with appropriate training for medical students and medical teachers, and the promotion of workshops and different exposures.

Concerning the feasibility of our curriculum, this intervention is simple and performed by a trained professor, in traditional classrooms and for large groups of students. This intervention, which takes $2 \mathrm{~h}$ a week, can be incorporated even in curricula with lack of space and the techniques applied are easy to administer and based on the well-known evidencebased work of Kabat-Zinn. ${ }^{10}$ Nevertheless, due to our nonsignificant results, some adaptations should be considered. As reported below, more studies are needed to evaluate students in a long term and in different moments of the medical training. Likewise, a longer intervention could potentially improve students' awareness of the technique, allowing them to use it in exam periods and stressful moments throughout the course. We believe this curriculum could be further tested in international medical schools in order to see if there is a difference in other medical training contexts and cultural backgrounds.

This work presents some limitations that must be mentioned. First, in spite of following students' class attendance, we do not have a precise data on how many students engaged with and practiced mindfulness. The development of a mindfulness diary was attempted but student response was low (approximately $20 \%$ brought the meditation diary in the classes), and, thus, this instrument was discontinued. Second, researchers should be careful when generalizing these results because they are derived from only one Brazilian public education institution. Third, the study was done during the course's first weeks when few assessments had taken place. We do not know if these results and the use of mindfulness would be different if students were followed during the entire semester. In the final weeks of the semester, the final exams usually are concentrated, which could lead to higher levels of distress in these students. In order to minimize this suffer, students may use more frequently this technique. Forth, our follow-up period was short and a longer follow-up could result in different outcomes. Finally, although we have taken some actions trying to reduce the sharing of information between students (classes were in different floors of the medical school building and we have asked them to not tell their classmates about the intervention), it is difficult to quantify how the crosscontamination between groups could have influenced our results. Even with these limitations, a positive characteristic our study presents is that it is a randomized controlled trial in which baseline groups were similar, minimizing the impact of being volunteers or not and the methodological problems of quasiexperimental studies.

In conclusion, the incorporation of a required mindfulness course for large groups in the curriculum during the first semester of medical training was not associated with an improvement on medical students' mental health and quality of life. Apparently, beyond proving the benefits and development of the state mindfulness bring and the differences in the methods of teaching in and of itself, there is a need to identify which groups are responsive to the method, bearing in mind that this is not a passive intervention, since It demands incorporating a change in life habits by the individual.

Corresponding Author: Giancarlo Lucchetti, $M D, P h D$; School of Medicine Federal University of Juiz de Fora, Juiz de Fora, MG, Brazil (e-mail: g.lucchetti@yahoo.com.br).

\section{Compliance with Ethical Standards:}

The study was approved by the UFJF University Hospital's Ethics in Research Committee.

Conflict of Interest: The authors declare that they do not have a conflict of interest.

\section{REFERENCES}

1. Dyrbye LN, Thomas MR, Shanafelt TD. Systematic review of depression, anxiety, and other indicators of psychological distress among US and Canadian medical students. Acad Med. 2006;81(4):354-73

2. Ghodasara SL, Davidson MA, Reich MS, Savoie CV, Rodgers SM Assessing student mental health at the Vanderbilt University School of Medicine. Acad Med. 2011;86(1):116-21.

3. Moutinho ILD, Maddalena NdCP, Roland RK, Lucchetti ALG, Tibiriçá SHC, Ezequiel OdS, et al. Depression, stress and anxiety in medical students: a cross-sectional comparison between students from different semesters. Rev Assoc Méd Bras. 2017;63(1):21-8. 
4. Rotenstein LS, Ramos MA, Torre M, Segal JB, Peluso MJ, Guille C, et al. Prevalence of depression, depressive symptoms, and suicidal ideation among medical students: a systematic review and meta-analysis. JAMA. 2016;316(21):2214-36.

5. Bassols AM, Okabayashi LS, Silva ABd, Carneiro BB, Feijó F, Guimarães GC, et al. First-and last-year medical students: is there a difference in the prevalence and intensity of anxiety and depressive symptoms? Rev Bras Psiquiatr. 2014;36(3):233-40.

6. Kötter T, Tautphäus $\mathbf{Y}$, Obst KU, Voltmer E, Scherer M. Healthpromoting factors in the freshman year of medical school: a longitudinal study. Med Educ. 2016;50(6):646-56.

7. dos Santos Boni RA, Paiva CE, de Oliveira MA, Lucchetti G, Fregnani JHTG, Paiva BSR. Burnout among medical students during the first years of undergraduate school: Prevalence and associated factors. PloS One. 2018;13(3):e0191746.

8. Lopes CS, Abreu GdA, Santos DFd, Menezes PR, Carvalho KMBd, Cunha CdF et al. ERICA: prevalence of common mental disorders in Brazilian adolescents. Rev Saude Publica. 2016;50:14s.

9. Khoury B, Sharma M, Rush SE, Fournier C. Mindfulness-based stress reduction for healthy individuals: a meta-analysis. J Psychosom Res. 2015;78(6):519-28.

10. Kabat-Zinn J. Mindfulness-based interventions in context: past, present, and future. Clin Psychol Sci Pract. 2003;10(2):144-56

11. Kabat-Zinn J. Full catastrophe living: Using the wisdom of your body and mind to face stress, pain, and illness 15th ed. New York: : Delta Trade Paperback.; 2005.

12. Demarzo M, Montero-Marin J, Puebla-Guedea M, Navarro-Gil $\mathbf{M}$, Herrera-Mercadal P, Moreno-González S, et al. Efficacy of 8-and 4session mindfulness-based interventions in a non-clinical population: A controlled study. Front Psychol. 2017;8:1343.

13. Shapiro SL, Schwartz GE, Bonner G. Effects of mindfulness-based stress reduction on medical and premedical students. J Behav Med. 1998;21(6):581-99.

14. Dobkin PL, Hutchinson TA. Teaching mindfulness in medical school: where are we now and where are we going? Med Educ. 2013;47(8):768-79.

15. Phang CK, Mukhtar F, Ibrahim N, Keng S-L, Sidik SM. Effects of a brief mindfulness-based intervention program for stress management among medical students: the Mindful-Gym randomized controlled study. Adv Health Sci Educ. 2015;20(5):1115-34.

16. Greeson JM, Toohey MJ, Pearce MJ. An adapted, four-week mind-body skills group for medical students: reducing stress, increasing mindfulness, and enhancing self-care. Explore J Sci Healing. 2015;11(3): 186-92.

17. de Vibe $\mathbf{M}$, Solhaug I, Tyssen $\mathbf{R}$, Friborg $\mathbf{O}$, Rosenvinge $\mathbf{J H}$, Sørlie $\mathbf{T}$ et al. Mindfulness training for stress management: a randomised controlled study of medical and psychology students. BMC Med Educ. 2013;13(1): 107 .
18. Warnecke E, Quinn S, Ogden $\mathbf{K}$, Towle N, Nelson MR. A randomised controlled trial of the effects of mindfulness practice on medical student stress levels. Med Educ. 2011;45(4):381-8.

19. Lucchetti G, Lucchetti ALG, Peres MF, Leão FC, Moreira-Almeida A Koenig HG. Validation of the duke religion index: DUREL (Portuguese version). J Relig Health. 2012;51(2):579-86.

20. Vignola RCB, Tucci AM. Adaptation and validation of the depression, anxiety and stress scale (DASS) to Brazilian Portuguese. J Affect Disord. 2014;155:104-9

21. Castro MMLD, Hökerberg YHM, Passos SRL. Dimensional validity of WHOQOL-BREF in health workers. Cad Saude Publica. 2013;29(7): 1357-69

22. Barros VVd, Kozasa EH, Souza ICWd, Ronzani TM. Validity evidence of the Brazilian version of the Five Facet Mindfulness Questionnaire (FFMQ). Psicologia: Teoria e Pesquisa. 2014;30(3):317-27.

23. McConville J, McAleer R, Hahne A. Mindfulness Training for Health Profession Students-The Effect of Mindfulness Training on Psychological Well-Being, Learning and Clinical Performance of Health Professional Students: A Systematic Review of Randomized and Non-randomized Controlled Trials. Explore (New York, NY). 2017;13(1):26-45.

24. Cohen J. A power primer. Psychol Bull. 1992;112(1): 155.

25. Slavin SJ, Schindler DL, Chibnall JT. Medical student mental health 3.0: improving student wellness through curricular changes. Acad Med. 2014:89(4):573.

26. Dyrbye LN, Shanafelt TD, Werner L, Sood A, Satele D, Wolanskyj AP. The impact of a required longitudinal stress management and resilience training course for first-year medical students. J Gen Intern Med. 2017;32(12):1309-14.

27. Aherne D, Farrant K, Hickey L, Hickey E, McGrath L, McGrath D. Mindfulness based stress reduction for medical students: optimising student satisfaction and engagement. BMC Med Educ. 2016;16(1):209.

28. Lucchetti G, Damiano RF, DiLalla LF, Lucchetti ALG, Moutinho ILD, da Silva Ezequiel O, et al. Cross-cultural differences in mental health, quality of life, empathy, and burnout between US and Brazilian medical students. Acad Psychiatry. 2018;42(1):62-7.

29. de Vibe M, Solhaug I, Tyssen R, Friborg O, Rosenvinge JH, Sørlie T, et al. Does personality moderate the effects of mindfulness training for medical and psychology students? Mindfulness. 2015;6(2):281-9.

30. Kuhlmann SM, Bürger A, Esser G, Hammerle F. A mindfulness-based stress prevention training for medical students (MediMind): study protocol for a randomized controlled trial. Trials. 2015;16(1):40.

31. Charlene Luchterhand M, Rakel D, Haq C, Grant L, Byars-Winston A. Creating a culture of mindfulness in medicine. WMJ. 2015.

Publisher's Note Springer Nature remains neutral with regard to jurisdictional claims in published maps and institutional affiliations. 